- Drug users have a special need for dental care.

- Oral health can be a low priority for them whilst using drugs.

- Drug users have the same entitlement as anyone else to dental care.

- Barriers to care include fear of dentists, needle phobia, the acceptability of dental services, the ability to self-medicate and organisational factors in their lifestyles.

- Dental care of recovering drug users can not only improve their oral health, but can assist them construct a 'non-addict' identity.

\title{
Drug users: oral health-related attitudes and behaviours
}

\author{
P. G. Robinson, ${ }^{1}$ S. Acquah ${ }^{2}$ and B. Gibson ${ }^{3}$
}

\begin{abstract}
Aim Explore oral health-related attitudes and behaviours of drug users. Design Qualitative study using focus groups and semi-structured interviews.

Setting Facilities for treatment and recovery of drug users in South London.

Participants Twenty-six male and 14 female recovering drug users. Results Participants described many changes in lifestyle associated with drug use including the physical effects of drugs, dietary habits, organisational and time constraints and unfavourable social conditions, which were not conducive to oral health. There was considerable health consciousness. They associated general health problems and problems with teeth, gums and oral soft tissues to both direct and indirect effects of drug use. Use of dental services was inhibited by low priority for oral health relative to the need to obtain and use drugs, experientially induced fear of dentists, the acceptability of dental services, needlephobia, ability to self-medicate and organisational factors in their lifestyles.

Conclusions The lifestyles of drug users may contribute to oral health problems and low use of services. Drug users therefore comprise a group with special dental needs and need greater access to dental care than most people. Much of this care could be provided in general practice where appropriate dental care can contribute to recovery from drug use.
\end{abstract}

A problem drug user can be considered as any person who experiences social, psychological, physical or legal problems related to intoxication and/or regular excessive consumption and/or dependence as a result of his or her own use of drugs or other chemical substances. ${ }^{1}$

There is a wealth of information on the epidemiology, medical complications and needs of drug users. ${ }^{2}$ Dental research has focused on its clinical implications and a number of authoritative reviews are available. ${ }^{3,4}$ Medical complications of drug use that are relevant to dentistry include abscesses at injection sites, viral

${ }^{1}$ Senior Lecturer, ${ }^{2}$ Post-Graduate Student, ${ }^{3}$ Lecturer. Division of Dental Public Health and Oral Health Services Research, GKT Dental Institute, King's College London

Correspondence to: Peter G. Robinson, Dept Oral Health and Development, School of

Clinical Dentistry, Claremont Crescent, Sheffield S10 2TA

Email: peter.g.robinson@sheffield.ac.uk

\section{Refereed paper}

Received 23.05.03; Accepted 10.02.04

DOI: $10.1038 /$ sj.bdj.4812090

(c) British Dental Journal 2005; 198: 219-224 hepatitis, Human Immunodeficiency Virus, endocarditis and anaesthesia complications. ${ }^{5}$

Tobacco smoking is an independent risk factor in periodontal disease and a major risk factor in oral cancers. ${ }^{6,7}$ The most prevalent diseases of the mouth in alcohol users include dental caries, traumatised teeth, periodontal diseases, cancer, recurrent oral ulceration, dermatoses and oral carcinomas. ${ }^{8}$

The oral health of users of other drugs has received less attention. There are case reports of specific oral changes although epidemiological research shows these changes to be uncommon. ${ }^{9}$ High caries experience is seen in heroin users, perhaps caused by a combination of xerostomia caused by opiates and the high sugar content of oral methadone solutions used to manage withdrawal from the drug..$^{9-13}$ Oral cleanliness may also be reduced in opiate users. ${ }^{13}$ Oral effects of cocaine are related to administration of the drug via nasal inhalation, smoking and direct smearing on the oral mucosa, especially the gingivae. ${ }^{14,15}$ Cocaine has a vasoconstrictive effect that causes ulceration and atropy of the tissues. There may also be stimulant effects on the facial and masticatory muscles.

Drug users may also have special needs in relation to receiving dental care. Anecdotally, they may be dentally anxious and have low pain tolerance requiring careful pain relief and a good rapport with the dentist. 5,16,17 They report difficulty accessing dental treatment, less frequent use of dental services and reluctance on the part of dentists to treat them. ${ }^{18-20}$ Similar difficulties have been noted in other areas of healthcare. Drug users experience marginalisation and avoidance by service providers and perceive services to be unavailable. ${ }^{21,22}$

The direct oral implications of drug use are complicated by other factors such as social background, the need to obtain funds for drugs and other lifestyle factors. Little dental research has considered these aspects of drug use. Some time ago drug-users were noted to be a socially deprived group whose erratic lives inhibited regular dental care with a high frequency of broken and cancelled appointments. ${ }^{23}$ Poor dental health seemed related to their lifestyle habits as much as the drugs.

Scheutz also explored attitudes and behaviours using semi-structured interviews with a group of Danish drug users in the 1980s. ${ }^{24}$ Participants were predominantly interested in obtaining pain relief for dental emergencies. Many neglected oral hygiene and had high sugar consumption. Their dietary habits were related to the injection of the drugs in a ritual manner. The drug users became ashamed of 
the appearance of their mouths during drug-free periods but felt that it did not hamper attempts at resocialisation. Not only were these data collected in another country, but, they are almost 20 years old. Oral health, the nature of drug use and attitudes to both oral health and drug use have changed considerably in the interim.

One reason why there is so little research from the perspective of the drug-users is that the factors that limit dental care also preclude participation in research. One group of people who are more accessible is recovering drug users. Thus this study aimed to provide insights into the oral health-related attitudes and behaviours of drug users from the perspective of those people who were currently recovering from problematic drug use.

\section{MATERIALS AND METHODS}

A qualitative approach was adopted because very little is known about the oral health-related knowledge, attitudes and behaviours of drug users in the UK.

The target population was recovering drug users accessing drug detoxification, recovery and rehabilitation units in South London. All participants were over 18 years and were not experiencing withdrawal symptoms. Each was receiving a specified amount of medication or was drug free. People who were dependent only on alcohol and/or benzodiazepines were not invited to take part. Potential participants were given information about the research and why it was being carried out, and were asked individually for their written permission to participate. All data were collected in the months of June and July 1999.

Data were collected in focus groups followed by individual semistructured interviews with other participants. Focus groups capitalised on interaction between participants to generate data whereas individual interviews allowed the field researcher (SA) to probe into private topics and to clarify issues that had emerged in the focus groups. This process allowed triangulation of the data by using a different approach and permitted the emergence of private accounts that participants may not wish to have revealed in focus groups. ${ }^{25}$ Qualitative research does not aim to provide data that are statistically generalisable from the study to a wider population, but aims to capture the range of views and experiences. For this reason data collection continued until saturation, when no new themes emerged.

Between four and eight people participated in each of four focus groups (25 people in total). Two focus groups were conducted on an acute drug detoxification unit, one on a recovery unit and another on a drug rehabilitation unit. All the discussions were conducted in the evenings, and in relaxed and comfortable settings, avoiding interference with formal activities and minimising interruptions. The topics included daily routines, personal and oral hygiene, dietary habits, general health, drug use, oral health problems, methods of oral and general pain relief, help-seeking behaviour and experience with dentists.

Fifteen other recovering drug users recruited from acute detoxification and recovery wards took part in semi-structured interviews. The interviews were guided by a preliminary content analysis of the focus group discussions. Each interview lasted 45 to 50 minutes.

Data were recorded onto audio-tape and transcribed. The aim of the data analysis was to develop the details of the major themes around which the data collection was conducted. It was thought that these aspects of the data would be of direct clinical relevance to dentists. Transcripts and the moderator's notes were subject to content analysis by coding. Original codes were assigned to mark themes identified in the transcripts that were relevant to the area of enquiry. Related codes were then grouped together as categories that emerged from the data, these were subsequently linked in analytical categories. ${ }^{25}$ In order to satisfy the criteria for reliability and validity, all three authors reviewed the data. Sufficient data to provide a life context for the participants is reported but the focus is predominantly on oral health. The data are presented arranged by the analytical categories that emerged from the data and quotes are used (with pseudonyms) to illustrate the categories and to typify statements as a form of validation.

The study was approved by the Local Research Ethics Committee of the Merton, Sutton and Wandsworth Health Authority and by the Research Ethics Committee of King's College London.

\section{RESULTS}

Forty recovering drug users took part. Their ages ranged from 21 years to 52 years and 26 were men. All the participants were British, except one female from Mozambique. Twenty-seven were in a detoxification programme, nine were in a recovery unit following completion of detoxification and four were in a residential drug rehabilitation unit.

Participants had used drugs for between eight and 38 years. Most participants had used heroin and methadone, with many using alcohol and other drugs in addition. Others used predominantly stimulants such as cocaine and its derivatives and amphetamines. All the participants smoked tobacco.

All the participants had formal education, most up to the age of 16 years. Six had gone on to higher or further education. Several described unhappy experiences at school such as not getting on with friends, being shy, lacking confidence or truanting by themselves or with friends. Others had not been in mainstream schools. Employment history varied. Only two participants were in formal employment at the time of the investigation. They had taken time off work to engage in treatment. Most had previous work experience, which included mainly unskilled jobs such as rubbish clearing or painting and decorating. One had no previous work record. Most participants rented accommodation, a few were facing eviction due to rent and council tax arrears. The remaining participants did not have a place they called their own, but were staying with friends or family. One described his predicament as:

'I lost my flat. I had nothing, I lost my job, I just went from bad to worse and I didn't really care until I hit rock bottom. And when I hit rock bottom I couldn't go down any further. I was like waking up in places I've never been before. I didn't know where I was, like park benches. Like I'd wake up in parks and on park benches, shop doorways and things like that.'

(Adrian, 7/7/99, Interview)

In describing the state of accommodation of a drug user, one of the participants commented:

'There's syringe packets on the floor, dirty ash trays all over the place, plates on the draining board with all dirty food and mould on and everything like that.'

(Richard, 30/6/99, Interview)

Sometimes homelessness affected oral hygiene. It was only possible if they had sheltered accommodation, with running water to brush their teeth. Participants also reported that oral hygiene would be ignored if they went on a binge or went into a "crack house' for a number of days. This was because they were either 'out of it' or would find themselves in someone's house, and without their toothbrush.

All of the participants felt that harm to them from drug use outweighed the benefit:

'It (drug use) temporarily destroyed my family, wrecked my family, in their tender years as well, you know 11, ten and a five-year-old. And all these quality time and quality years have been wasted, like with me in treatment for a year.'

(Jenny, 7/7/99, Interview) 


\section{Avoiding withdrawal}

A primary concern during drug use had been to avoid symptoms of withdrawal, described as being 'ill' or 'sick'. Participants felt strongly about withdrawal as the worse kind of experience they went through.

'I mean very ill. It's like having a very bad bout of flu, but nine or ten times worse, you know. I mean stomach cramps, the headaches, the lack of sleep. I say lack of sleep like no sleep, diarrhoea, bladder problems, fatigue, not having the energy to sort of do the things you should normally do, like getting up and have a shower, wash or anything.

(Callum, 8/6/99, Focus Group)

Participants had to prevent the effects of withdrawal vigorously in a sequence of raising money, finding a dealer, purchasing the drug and using it. Getting up in the morning usually meant experiencing withdrawal symptoms because few participants were able to save drugs for the morning. Users had to dress quickly 'to go out and get gear'. The most important thing was to 'dress smartly in order not to attract attention'. Personal hygiene was neither important nor necessary and it went downhill dramatically.

'I mean having a habit is like having a full-time job, you know. It's like getting up in the morning. If you have to steal, you go out earning the money, if you go out stealing, you get the stuff, you spend most of the day trying to sell it and when you do sell it you're looking at the rest of the day trying to get heroin. And then you've got to find somewhere to take it.'

(Tracey, 18/7/99, Interview)

Having walked 'round and round' throughout the day meant coming home tired. The drug user then had a 'hit' and 'gouged out' without seeing to personal hygiene. On waking up the cycle repeated itself.

Other participants also explained that they neglected their oral and personal hygiene because of low self-esteem.

'Hygiene is the last of your worries I didn't care about myself at all. I didn't give a shit. I really didn't care, fucked up everything, kids, girlfriend, family, myself. Felt I wasn't worth anything because of what I was.'

(Graham, 12/6/99, Focus group)

The few who were able to maintain themselves and their selfcare did so with extraordinary effort unless they had regular supply of drugs to keep withdrawal symptoms at bay.

Those who reported brushing their teeth said they would 'do it really quick, in less than a minute'. Sometimes putting the brush in the mouth would cause the drug user to 'start heaving and feeling sick'. Only the few who were quite determined attempted to try again once they 'stop heaving'.

\section{Drug use and health}

Participants were acutely aware of their health. Many held the view that drug use:

'Takes all the natural goodness out of their bodies, destroying their immunity, breaking down the defence system that keeps the body healthy.'

(Stephen, 10/6/99, Focus group)

The effects of smoking were minimised by the participants. The only concerns were a shortness of breath and coughing. Many stated that smoking would be the last thing they would consider giving up as they felt they were already sacrificing enough by giving up illicit drug use. Some had bought tooth whitening paste or baking soda to remove smoking stains. Others had used very hard bristle toothbrushes, one of which had caused teeth to be 'sheared off at the back over a period of time'.

\section{Drug use and oral health}

All the participants described 'rotten teeth', abscesses, toothache and tooth loss and blamed methadone linctus to some extent for their dental decay. They attributed this effect to the sugar content, its stickiness and because it gave them a sweet tooth. Some participants observed that using heroin for a number of years kept their teeth in a reasonable state, but they noticed pain all over the mouth soon after starting methadone. Others felt that a combination of factors came into play:

'But its [tooth decay] always to do with the heroin, I think the citric acid, and the methadone that we take and the stage you get when you don't look after your hygiene.'

(Diane, 8/6/99, Focus group)

Participants also described the numbing effect of specific drugs on the mouth:

'The first thing I do when I get a bag of cocaine is to test the strength of it. I wipe it on my finger, rub it in my gum and I can tell instantly. I mean it numbs your gum instantly. You can tell the strength by how quickly the numbness spreads.'

(Graham, 12/6/99, Focus group)

Stimulants were said to make the teeth 'wobble and feeling like you can rip them out'. Participants commented that stimulants caused constant grinding or clenching with accompanying crunching noises in the ear. The consequences of this activity were sensitive and worn teeth followed by difficulty opening the jaw or chewing because of pain. This movement was also reported to damage the tongue, cheeks and lips.

Many participants reported bleeding [gums], receding gum line, bad breath and 'rotten gums' but made the observation that tooth brushing reversed the process.

A number of participants said that they frequently ended up in hospital casualty departments because of accidents. Breaking or losing teeth during accidents and fights further compromised the oral health of participants.

\section{Diet}

Without exception, participants acknowledged that their diets were unhealthy and were a consequence of a chaotic life. They said that their drug use suppressed their appetites, but also caused them to crave for sugar. Some felt that their stomachs had shrunk, making it hard for them to eat proper food. Drugs, especially opiates, affected their taste sensation, making ordinary meals taste like 'cardboard', so that they could not appreciate food. Their mouths and sense of smell were constantly numbed by drug use.

Several participants had craved sugar and consumed huge amounts in various forms. A 'typical diet' might consist of biscuits, ice cream, yoghurt, doughnuts and confectionary with between three and eight teaspoonfuls of sugar in their beverages.

Sugary foods provided participants with the energy to pursue their full-time occupation of drug use and had to be something quick and easy to be consumed on the move. Approaches to preparing meals varied considerably. Cooking was often seen as a time consuming activity that required skill and facilities. Several participants considered cooking to be dangerous while on drugs because they might fall asleep in the process. Even those who prepared meals for their family lacked an appetite themselves and 
reported losing weight. Moreover, participants did not have the money to spare on cooking, because:

'It's like all your money goes into your drug habit, you know. When you go shopping for food, that's secondary. It's like you put that last.'

(John B, 10/6/99, Focus group)

Participants' eating habits and body weight improved once they got into treatment or rehabilitation programmes or maintained a drug-free lifestyle. They had time to sit and eat, their appetites and sense of taste improved whereas nausea, vomiting and constipation resolved.

'I can taste food now. I can taste the meat, vegetables and I enjoy my food more and I appreciate my food more.'

(Jason, 10/6/99, Focus group)

\section{Health-seeking behaviour}

Many participants had lived in a state of denial about the impact of their drug use, waiting until the last desperate moment before seeking help from elsewhere. Many participants had ignored health problems until they gained a place in a treatment or rehabilitation unit. Many avoided dentists until the pain became impossible to control.

Most had self-medicated for pain (especially dental pain). They would take extra drugs for severe toothache including methadone, dihydrocodeine, ibuprofen, heroin and cocaine; in some cases injected directly into a tooth or gums. Some participants had even removed teeth themselves in this way.

Participants' perception of dentists depended on such factors as treatment experience, NHS versus private care, intensity of drug use and the ability to keep appointments. Several described negative experiences and were 'petrified' of dentists. Only a minority were registered with a dentist. Others would only be comfortable if someone attended with them and most preferred general anaesthesia for dental treatment. Some even reported getting up from the dental chair and running away because they could not accept treatment.

One particular fear among participants was needle phobia.

'Horrible, horrible. I didn't like it, especially that big needle they put in your mouth. I hate it'

(Callum, 8/6/99, Focus group)

The drug using lifestyle also prevented dental attendance. Drug users identified their preoccupation with avoiding withdrawal, homelessness, prolonged bingeing, waiting lists for drug treatment and low self-esteem as barriers:

'You don't have time going to the dentist because it's the last thing you think of. All you think of is scoring your drugs. Once you've got your drugs you just want to chill out, you don't want to go to the dentist to be prodded about.'

(Ray, 12/7/99, Interview)

Even when dentists accepted them as patients, they were unable to attend or complete a course of treatment:

'Say, for example, I've been up all night with toothache, I'd phone and make an appointment. I wake up in the morning and if I've got no gear I'm sick. The appointment is at 10 o'clock. If I've not got gear with me by 10 o'clock, I'm not going to the appointment. I'm still out getting the money to buy my drugs in case I feel more ill on the gear. Or that I've had so much gear the day before, I'll wake up late.'

(Heather, 15/7/99, Interview)
Some concerns voiced about dentists were not related to drug use. One participant reported that dentists did not want to take on NHS patients and that the nearest NHS dentist was miles away from where they lived. Others were aware that dentists were paid for each item of service and felt that they might undertake work that wasn't necessary.

However, most participants reported feeling comfortable with dentists if the approach and procedure was right:

'She is brilliant with me. She put a temporary filling in my teeth to take away the pain. She sat down, calmed me down, told me what she was going to do. She put me at ease. She is really good that girl, I mean I'm not frightened of what she does.'

(Diane, 8/6/99, Focus group)

They also commented that by constantly talking to them, their minds were kept off what was being done and they were encouraged to maintain appointments. Participants were also aware that going to the dentist was not only treatment oriented but included receiving oral hygiene instruction and dietary advice.

Some participants felt that their drug use added to the difficulties of getting a dentist to treat them. They felt alienated because dentists placed them at the end of the day or worn extra gloves. Others reported hiding their drug use from dentists because they felt they would be treated differently.

Several participants highlighted the importance of oral health behaviours during their recovery from drug use.

'It's made me think, right I want to keep fit now, keep ...I want to do that, get out and train a couple of times a week and look after my teeth and that because I've never looked at them in the last couple of years and now all the feeling has come back in my body.... I can feel it now, this bottom gum hurts here. I'm gonna get all that sorted out, get it done.'

(Callan, 10/7/99, Interview)

\section{DISCUSSION}

In this study recovering drug users recalled that their over-riding concern had been to avoid withdrawal symptoms. Everything had been subservient to this priority. Participants reported high levels of oral disease and described behaviours that threatened oral health. Drug use is only one of many factors involved in the aetiology of dental disease in this group. A combination of low saliva secretion, increased consumption of sugars, unemployment and social exclusion are important considerations. These factors occur in addition to the medical complications of drug use. ${ }^{5}$

The apparently paradoxical relationship between drug use and acute health awareness might be explained by the nature of the sample. Participants were in health settings and were recovering from drug use. Moreover, the recovery from drug use can be seen as the reconstruction of a non-addict identity; a process that might involve reconstruction of the body with its associated focus on health. ${ }^{26}$ In order to do so the recovering user has to develop a range of new activities and relationships to sustain their new identity. It is at this point that the reconstruction of the mouth and teeth can become of central importance.

Many participants attributed their dental disease to methadone use. Oral methadone is commonly prescribed for short-term detoxification and long-term maintenance of opiate-dependent patients. The aim is to encourage users to switch from injecting to oral administration. ${ }^{27}$ The sugar content may be as much as $50 \% \mathrm{~m} \mathrm{v}^{-1}$ and some users hold the medication in the mouth although that was not the case with these participants. ${ }^{12}$ There are no longitudinal data to establish how much methadone alone contributes to dental caries. It is possible that the contribution of methadone to dental caries is a small part of a larger process. The cariogenic diets, 
xerostomia, decreased oral hygiene (with concomitant reduced exposure to fluoride) and lack of consideration of health during periods of drug use are likely to have contributed to the development of caries. ${ }^{12,13}$ In addition, symptoms of caries may have been masked by pharmacological effects. On occasions where symptoms were more severe, participants may have self-medicated.

There is anecdotal evidence of sugar craving among drug-users and high sugar consumption has been noted. ${ }^{28}$ However, the view that drug users crave sugar can also be interpreted as a number of factors acting together. Nausea, vomiting and appetite suppression will affect the intake and retention of food by chronic drug users. ${ }^{29}$ Furthermore, participants described effects on taste sensation that made food unpalatable. Despite their lack of appetite, obtaining drugs required considerable industry. Participants needed quick and easy snacks to be eaten on the go. High consumption of sugars, could be a necessity rather than a craving and would be a major aetiological factor for the high levels of dental caries seen in drug users. ${ }^{9,13}$

Dental problems may have become more noticeable during methadone use because it is less sedative and does not have the same analgesic effect as heroin. Withdrawal symptoms may also exacerbate any existing dental problems. Moreover, recovering drug users taking methadone were not preoccupied with obtaining opiates and their dental health may have had a greater impact on them. These hypotheses are compatible with the data and could be an avenue for future research.

Plaque removal by oral hygiene procedures was directly inhibited by drug use and indirectly by the associated lifestyle and low self-esteem. With a combination of poor oral hygiene, tobacco smoking, the local effects of other drugs and bruxism the risk of periodontal diseases may be higher for drug users. These findings are compatible with the few epidemiological data on oral cleanliness and periodontal changes. ${ }^{13}$

Participants reported a range of traumatic episodes including grinding associated with toothwear, damage to the oral soft tissues and symptoms of temperomandibular joint disorders. The use of stimulants has been associated with bruxism and excessive toothwear in the past. ${ }^{10,30}$ In addition, fights or accidents threatened oral health. Drug intake increased to cope with the pain, but the analgesic effect may have masked the damage in some instances, so delaying appropriate intervention.

Participants identified factors both related and unrelated to drug use as barriers to care. Factors not directly related to drug use included low availability of dental care and fear of dentists. These factors are common to many people (for a review see Kent and Croucher ${ }^{31}$ ). It seems ironic that drug users who self-inject on a daily basis should be deterred from attending the dentist because of a fear of needles. Dental anxiety was measured in a small sample of methadone users in Scotland, half of whom had scores that were strongly suggestive of phobia. ${ }^{32}$ In addition, most people who experience problems with drug use are already marginalised and excluded. Therefore, whether or not drug users are truly phobic, they often have difficulties accepting the responsibility and obligations of obtaining dental treatment.

Drug related factors including homelessness, prolonged bingeing on drugs, waiting lists for drug treatment and rehabilitation units, low self-esteem and the poor acceptability of services all acted as additional barriers to care.

These data indicate that drug users require special dental care. In addition to having high rates of dental disease $\mathrm{e}^{9,13}$ and commensurate needs, they have difficulty accessing services. With their reluctance to visit the dentist and a greater likelihood of selfmedication, dentists have an important role to play in prevention of exacerbated drug use. Drug users therefore need greater access to dental care than most people.

This need does not imply that they should be seen by specialists. Dental care could be provided in general dental practice, as the management of dental disease in drug users does not typically require specialist knowledge or expertise. Department of Health policy throughout the United Kingdom is explicit that drug misusers have the same entitlement as other patients to the services provided by the National Health Service. ${ }^{33}$ There is a responsibility to provide care for both general health needs and drug-related problems. These guidelines stress the importance of a shared care approach as a rational model to improve service delivery, using different skills and covering a range of treatment options.

Traditional patterns of dental registration with dentists who are paid on a fee-per-item basis are not suitable for drug users. Dentists may be unwilling to accept drug users whom they fear may not complete treatment causing them to lose valuable time and income. Even services dedicated to drug users tend to opt for less complex treatment for similar reasons. ${ }^{34}$ During periods of drug use these participants had only been concerned about treatment for pain relief. Again, this type of emergency attendance is not compatible with registration with a dentist. Likewise, those users who were homeless constantly moved from one place to another, again reducing the chance of accessing local services on a regular basis. ${ }^{35}$ Finally, the calm and careful approaches to which participants responded is an aspect of quality that is most likely to be lost under existing arrangements. All of these difficulties may be overcome by new contractual arrangements such as dental access centres or other locally planned services where care is not provided under fee-per-item only to registered patients. ${ }^{36,37}$

Drug users also reported 'hiding' their drug use from dentists ${ }^{38}$ and other health professionals because of fear of being treated differently or being refused treatment. Similar behaviour has been noted among other patient groups who find services unacceptable. ${ }^{39}$ These data indicate that it is self-defeating for dentists to deny care on the grounds of patients' medical histories. The principal consequence is simply to reduce communication between patient and dentist.

Drug users may not be able to take full control of their lives and health until they are able to enter a place of safety, which in most cases is a drug treatment and rehabilitation unit. ${ }^{40}$ Removing the need to take drugs helps them to focus more on their health. The facilities available, the attention received, and the support of staff and fellow drug users bolster self-esteem and motivate self-care. It follows that patients who are in rehabilitation should be given easy access to dental care. Indeed, if dental treatment plays a role in the reconstruction of a non-drug using identity it will contribute to their recovery from drug use. ${ }^{26}$ Drug rehabilitation units often have referral systems to health and other services that aim to improve the overall health of drug users. Dentists should participate in these networks whenever invited. In this way dentists can act in line with UK Government strategy for tackling drug misuse. ${ }^{41}$ The strategy emphasises the need for drug users to have proper access to appropriate services, including primary care, which should support them in changing their behaviour towards more positive lifestyles.

The authors express their gratitude to the participants who gave their time and shared their experiences for this research. They would also like to thank the staff of the clinics for allowing the data collection to take place. Finally the authors wish to acknowledge the support of colleagues in the Division of Dental Public Health at the GKT Dental Institute who commented on earlier drafts of this manuscript.

1. Advisory Council on the Misuse of Drugs. AIDS and drug misuse (part 1). London: HMSO; 1988.

2. Fountain J, Griffiths P. Synthesis of qualitative research on drug use in the European Union: Report on an EMCDDA Project. Eur Addict Res 1999; 5: 4-20.

3. Lee $C Y$, Mohammadi $H$, Dixon R A. Medical and dental implications of cocaine abuse. J Oral Maxillofac Surg1991; 49: 290-293.

Rees T D. Oral effects of drug abuse. Crit Rev Oral Bio/ 1992; 3: 163-184.

5. Meechan J G. Drug abuse and dentistry. Dental Update 1999; 26: 182-187, 190.

6. Johnson N M, Warnakulasuriya K A A S. Epidemiology and aetiology of oral cancer in the United Kingdom. Commun Dent Health 1993; 10: 13-29. 
7. Stoltenberg J L, Osborn J B, Pihlstrom B L, Herzberg M C, Aeppli D M, Wolff L F, Fischer $G$ E. Association between cigarette smoking, bacterial pathogens, and periodontal status. J Periodontol 1993; 64: 1225-1230.

8. Gelbier S, Harris C. Oral and dental health in the alcohol misuser. Addiction Biol 1996; 1: 165-169.

9. Du M, Bedi R, Guo L, Champion J, Fan M, Holt R. Oral health status of heroin users in a rehabilitation centre in Hubei province, China. Commun Dent Health 2001; 18: 94-98.

10. Rosenbaum C H. Did you treat an addict today? Int Dent J 1981; 31:30-312.

11. Angelillo I F, Grasso G M, Sagliocco G, Villari P, D'Errico M M. Dental health in a group of drug addicts in Italy. Commun Dent Oral Epidemiol 1991; 19:36-37.

12. Sheedy J J. Methadone and caries. Case reports. Aus Dent J 1996; 41:367- 369 .

13. Molendijk B, Ter Horst G, Kasbergen M, Truin G J, Mulder J. Dental health in Dutch drug addicts. Commun Dent Oral Epidemiol 1996; 24: 117-119.

14. Parry J, Porter S, Scully C, Flint S, Parry M G. Mucosal lesions due to oral cocaine use. BrDent J 1996; 180: 462-464.

15. Kapila Y L, Kashani H. Cocaine-associated rapid gingival recession and dental erosion. A case report. J Periodontol 1997; 68: 485-488.

16. Ratcliff J S, Collins G B. Dental management of the recovered chemically dependent patient. JAm Dent Assoc 1987; 114: 601-603.

17. Sainsbury D. Drug addiction and dental care. NZ Dent J 1999; 95: 58-61.

18. Dawkes M, Sparkes S, Smith M. Dentists' responses to drug misusers. Health Trends 1995; 27: 12-14.

19. Sheridan J, Aggleton M, Carson T. Dental health and access to dental treatment: a comparison of drug users and non-drug users attending community pharmacies. $\mathrm{Br}$ Dent J 2001: 191: 453-457.

20. Metsch L R, Crandall L, Wohler-Torres B, Miles C C, Chitwood D D, McCoy C B. Met and unmet need for dental services among active drug users in Miami, Florida. $J$ Behav Health Serv Res 2002; 29: 176-188.

21. Travers R, Schneider M. Bparriers to accessibility for lesbian and gay youth needing addiction services. Youth Sociol 1996; 27: 356-378.

22. Ortiz A, Soriano A, Galvan J, Rodriquez E, Gonzalez L, Unikel C. Characteristics of cocaine users their perception and their attitude towards treatment services. Salud Ment 1997: 8-14.

23. Scheutz F. Five-year evaluation of a dental care delivery system for drug addicts in Denmark. Commun Dent Oral Epidemiol 1984; 12: 29-34.

24. Scheutz F. Dental habits, knowledge, and attitudes of young drug addicts. Scand J Social Med 1985; 13: 35-40.
25. Pope C, Mays N. Qualitative research in health care. London: BMJ Publishing; 2000

26. McIntosh J, McKeganey N. Addicts' narratives of recovery from drug use: constructing a non-addict identity. Soc Sci Med 2000; 50:1501-1510.

27. Ghodse H. Drugs and addictive behaviour. a guide to treatment. 2nd ed. Oxford Blackwell Scientific Publication, 1995.

28. Zador D, Lyons Wall P M, Webster I. High sugar intake in a group of women on methadone maintenance in south western Sydney, Australia. Addiction 1996; 91: 1053-1061.

29. Rawson RA, Ling W. Opioid addiction treatment modalities and some guidelines to their optimal use. J Psychoactive Drugs 1991; 23: 151-163.

30. Redfearn P J, Agrawal N, Mair L H. An association between the regular use of 3, 4, methylenedioxy-metamphetamine (ecstasy) and excessive wear of teeth. Addiction Biol 1998; 93: 745-748

31. Kent G, Croucher R. Achieving oral health. 3rd ed. Oxford: Wright, 1998.

32 Cunningham $\mathrm{C}$, MacPherson A. The social impact of oral health and level of dental anxiety in a population of drug misusers in Edinburgh (abstract). Commun Dent Health 2001; 18: 119.

33 Department of Health Scottish Office Department of Health, Welsh Office Department of Health and Social Services, Northern Ireland. Drug misuse and dependence-guidelines on clinical management. London: HMSO; 1999.

34 ter Horst G. Molendijk B, Brouwer E Verhey H G. Differences in dental treatment plan and planning for drug-addicted and non-drug-addicted patients. Commun Dent Ora Epidemio/1996; 24: 120-123.

35. Edwards D M, Watt R. Oral health care in the lives of gypsy travellers in East Hertfordshire. Br Dent J 1997; 183: 252-257.

36. Department of Health. Modernising NHS Dentistry. Implementing the NHS plan London: The Stationery Office; 2000.

37. HMG. The Queen's Speech. 2002.

38. McDaniel T F, Miller $D$, Jones $R$, Davis M. Assessing patient willingness to revea health history information. J Am Dent Assoc 1995; 126: 375-379.

39. Robinson P G, Zakrzewska J, Maini M, Williamson D, Croucher $\mathrm{R}$. The dental visiting behaviour and experiences of men with HIV infection. Br Dent J 1994; 176: 175-179.

40. Ettore B. A study of voluntary drug agencies: their roles in the treatment and rehabilitation field. Br J Addiction 1987: 82: 681 - 689

41. Drugs Strategy Directorate. Tackling drugs to build a better Britain - the Government's ten-year strategy for tackling drugs misuse. London: The Stationery Office; 1998. 